\title{
A PROJECTIVE CHARACTERIZATION FOR SKT-MODULES
}

\author{
BRIAN D. WICK
}

\begin{abstract}
In this paper a class of abelian groups (SKT-modules), which includes the torsion totally projective groups, $S$-groups, and balanced projectives is shown to be a subclass of a projective class of groups with respect to a naturally defined class of short exact sequences called the ch-projective modules and ch-pure sequences, respectively. Every $Z_{p}$-module has a ch-pure projective resolution and every reduced ch-projective module is a summand of a SKT-module. It is finally shown that a $Z_{p}$-module $M$ is ch-projective if and only if, for every ordinal $\alpha$, the two $Z_{p}$-modules $p^{\alpha} M$ and $M / p^{\alpha} M$ are both ch-projective.
\end{abstract}

1. Definitions. If $p$ is a prime number then the ring of all rational numbers $a / b$ with $b$ relatively prime to $p$ will be denoted by $Z_{p}$.

The category of $Z_{p}$-modules are those abelian groups with the property that multiplication by a prime other than the prime $p$ is an automorphism of the group. If $M$ is a $Z_{p}$-module then (i) the torsion submodule of $M$ is the maximal torsion subgroup of $M$; (ii) $M$ is torsion-free if and only if the torsion submodule of $M$ is (0); (iii) $M$ is reduced if and only if $M$ is a reduced group; and (iv) $M$ is divisible if and only if $M$ is a divisible group.

$Z$ and $Q$ will denote the groups of integers and rational numbers respectively. The group $\operatorname{Ext}\left(Q / Z_{p}, *\right)$ will be denoted by $c(*)$.

The limit ordinal $\lambda$ is a limit ordinal cofinal with $\omega$ if there is a sequence of smaller ordinals $\beta_{i}$ such that $\lambda=\sup \beta_{i}$. Otherwise $\lambda$ is said to be not cofinal with $\omega$.

The family of vector spaces $K\left(p^{\lambda}, G\right)$ is defined for every group $G$ to be $p^{\lambda} c\left(G / p^{\lambda} G\right) / p^{\lambda+1} c\left(G / p^{\lambda} G\right)$ where $\lambda$ is any ordinal and $p$ is a prime.

Warfield [1] and [2] introduced two classes of groups, balanced projectives and $S$-groups, which will play an important role in this paper. For the limit ordinal $\lambda$, a $Z_{p}$-module $M$ is a $\lambda$-elementary balanced projective if and only if $p^{\lambda} M \simeq Z_{p}$ and $M / p^{\lambda} M$ is a totally projective $p$-group. The torsion subgroup of $M$ is called a $\lambda$-elementary $S$-group. A $Z_{p}$-module $M$ is a balanced projective if and only if is isomorphic to the direct sum of a totally projective $p$-group and $\lambda$-elementary balanced projectives for various limit ordinals $\lambda$. The torsion subgroup of a balanced projective is called an $S$-group.

A module $M$ is an SKT-module if and only if there is a balanced projective $K$ and an $S$-group $S$ such that $M$ is isomorphic to $K \oplus S$.

Received by the editors May 21, 1979 and, in revised form, October 8, 1979.

AMS (MOS) subject classifications (1970). Primary 13C10; Secondary $13 \mathrm{CO5}$. 
2. ch-projectives. Since the objective is to find a projective characterization for the SKT-modules; for the present, a short exact sequence $e: 0 \rightarrow A \rightarrow B \stackrel{\pi}{\rightarrow} C \rightarrow 0$ is said to be an SKT-pure sequence if for every SKT-module $M$, the natural map $\pi^{*}$ : $\operatorname{Hom}(M, B) \rightarrow \operatorname{Hom}(M, C)$ is surjective. It will be shown that a sequence $e:$ $0 \rightarrow A \rightarrow B \rightarrow C \rightarrow 0$ is SKT-pure if and only if the sequences $0 \rightarrow p^{\alpha} A \rightarrow p^{\alpha} B \rightarrow$ $p^{\alpha} C \rightarrow 0$ and $0 \rightarrow p^{\alpha} c(A) \rightarrow p^{\alpha} c(B) \rightarrow p^{\alpha} c(C) \rightarrow 0$ are exact for every ordinal $\alpha$.

THEOREM 2.1. If the sequence $0 \rightarrow A \rightarrow B \rightarrow C \rightarrow 0$ is SKT-pure then for every ordinal $\alpha$, the sequences $0 \rightarrow p^{\alpha} A \rightarrow p^{\alpha} B \rightarrow p^{\alpha} C \rightarrow 0$ and $0 \rightarrow p^{\alpha} c(A) \rightarrow p^{\alpha} c(B) \rightarrow$ $p^{\alpha} c(C) \rightarrow 0$ are exact.

Proof. Suppose that the sequence $e: 0 \rightarrow A \rightarrow B \stackrel{\pi}{\rightarrow} C \rightarrow 0$ is SKT-pure. It will first be shown that the natural maps $\pi: p^{\alpha} B \rightarrow p^{\alpha} C, \pi: p^{\alpha} B[p] \rightarrow p^{\alpha} C[p]$, and $\pi$ : $p^{\alpha} c(B) \rightarrow p^{\alpha} c(C)$ are all surjective for each ordinal $\alpha$ where for a group $G, G[p]$ is the set of elements with order $p$.

Let $\alpha$ be an ordinal and $\alpha=\lambda+n$ where $\lambda$ is a limit ordinal and $0<n<\omega$. Let $M$ be a $\lambda$-elementary balanced projective and $m$ a generator of the free $Z_{p}$-module $p^{\alpha} M$. If $c \in p^{\alpha} C$ then there is a homomorphism $f: p^{\alpha} M \rightarrow p^{\alpha} C$ such that $f(m)=c$. Since $f$ does not decrease heights, and $p^{\alpha} M$ is a $p$-nice submodule of $M$, and $M / p^{\alpha} M$ is a totally projective $p$-group; there is an extension $f^{*}: M \rightarrow C$ of $f[2$, 1.13]. Since the sequence $e$ is SKT-pure, there is a homomorphism $g: M \rightarrow B$ such that $\pi g=f^{*}$; furthermore, $g(m) \in p^{\alpha} B$ and $\pi(g(m))=c$. Thus $\pi: p^{\alpha} B \rightarrow p^{\alpha} C$ is surjective.

Similarly if $c \in p^{\alpha} c(C)$ then there is a homomorphism $f: M \rightarrow c(C)$ such that $f(m)=c$. Let $S$ be the torsion submodule of $M$ from which it follows that $f(S) \subseteq C$. Thus the restriction of $f$ to $S$ is a homomorphism from the $S$-group $S$ to $C$. There is a homomorphism $g: S \rightarrow B$ such that $\pi g=f: S \rightarrow C$. Since $M / S$ is torsion-free and divisible, $g$ lifts to a homomorphism $g^{*}: M \rightarrow c(B)$; furthermore, $\pi g^{*}=f: M \rightarrow c(C),[1]$. Since $g^{*}(m) \in p^{\alpha} c(B)$ and $\pi(g(m))=c$, the map $\pi$ : $p^{\alpha} c(B) \rightarrow p^{\alpha} c(C)$ is surjective.

Let $c \in p^{\alpha} C[p]$ and $H=M / p^{\alpha+1} M . H$ is a totally projective $p$-group such that $p^{\alpha} H$ is cyclic of order $p$. If $n$ is the generator of $p^{\alpha} H$ then there is a homomorphism $f: H \rightarrow C$ such that $f(n)=c$. There exists a homomorphism $g: H \rightarrow B$ such that $\pi g=f: H \rightarrow C$; furthermore, $g(n) \in p^{\alpha} B[p]$ and $\pi(g(n))=c$. Thus the map $\pi$ : $p^{\alpha} B[p] \rightarrow p^{\alpha} C[p]$ is surjective.

The desired result will follow from $[2,2.1]$.

Definition. A sequence $0 \rightarrow A \rightarrow B \rightarrow C \rightarrow 0$ is balanced if and only if the induced sequence $0 \rightarrow p^{\alpha} A \rightarrow p^{\alpha} B \rightarrow p^{\alpha} C \rightarrow 0$ is exact for every ordinal $\alpha$.

Definition. A sequence $0 \rightarrow A \rightarrow B \rightarrow C \rightarrow 0$ is ch-pure if and only if the sequences $0 \rightarrow A \rightarrow B \rightarrow C \rightarrow 0$ and $0 \rightarrow c(A) \rightarrow c(B) \rightarrow c(C) \rightarrow 0$ are balanced.

Definition. A module $M$ is ch-projective if and only if for every ch-pure sequence $0 \rightarrow A \rightarrow B \stackrel{\pi}{\rightarrow} C \rightarrow 0$, the natural map $\pi^{*}: \operatorname{Hom}(M, B) \rightarrow \operatorname{Hom}(M, C)$ is surjective.

THEOREM 2.2. Divisible modules and SKT-modules are ch-projective. 
Proof. If a sequence is ch-pure then it is balanced and by [1], divisible modules and balanced projectives are ch-projective.

To show that $S$-groups are ch-projective, only a $\lambda$-elementary $S$-group, $S$, needs to be considered. If $S$ is the torsion subgroup of the $\lambda$-elementary balanced projective $M$ then $M / S$ is torsion-free and divisible. Since the sequence $0 \rightarrow p^{\infty} A$ $\rightarrow p^{\infty} B \rightarrow p^{\infty} C \rightarrow 0$ is split exact, we may assume that $A, B$ and $C$ are reduced modules and submodules of their cotorsion envelopes $c(A), c(B)$ and $c(C)$ respectively. The fact that module $C$ is a submodule of $c(C)$ means that we can consider any homomorphism of $S$ into $C$ as a homomorphism from $S$ into $c(C)$; furthermore, $M / S$ being torsion-free and $c(C)$ being cotorsion implies that any homomorphism from $S$ into $c(C)$ can be lifted to a homomorphism from $M$ into $c(C)$. If $f: S \rightarrow C$ is a homomorphism then there is a homomorphism $f^{\prime}: M \rightarrow c(C)$ such that $f^{\prime}$ and $f$ agree on $S$. Since $M$ is a balanced projective module and the sequence $0 \rightarrow c(A) \rightarrow c(B) \rightarrow c(C) \rightarrow 0$ is balanced, there is a homomorphism $g: M \rightarrow c(B)$ such that $\pi g=f^{\prime}$. The map $g$ must send the torsion subgroup $S$ into the torsion subgroup of $c(B)$. Thus $g(S) \subseteq B$ and the restriction of $g$ to $S$ is a homomorphism from $S$ into $B$ such that $\pi g=f: S \rightarrow C$.

Theorems 2.1 and 2.3 imply that the class of SKT-pure sequences coincides with the class of ch-pure sequences.

One might conjecture that if either $e_{1}: 0 \rightarrow A \rightarrow B \rightarrow C \rightarrow 0$ or $e_{2}: 0 \rightarrow c(A) \rightarrow$ $c(B) \rightarrow c(C) \rightarrow 0$ is balanced then the other shall be balanced. However the following two examples show that both $e_{1}$ and $e_{2}$ must be balanced in order to show that $e_{1}$ is ch-pure. To show that $e_{1}$ being balanced does not imply that $e_{2}$ is balanced, take $C$ to be any $S$-group and $e_{1}$ a balanced projective resolution of $C$. By the conjecture, $e_{1}$ would be a ch-pure sequence; consequently it would be split exact; hence every $S$-group would be a totally projective group, which is not true. To show that $e_{2}$ being balanced does not imply that $e_{1}$ is balanced, take $A$ to be any reduced group, $B=c(A)$ and $C$ will be torsion-free and divisible. The sequence $e_{2}$ reduces to $0 \rightarrow c(A) \rightarrow c(B) \rightarrow 0$ which is balanced. The conjecture would imply that the sequence $e_{1}$ would be ch-pure and split. Thus $c(A)$ would not be reduced.

COROllary 2.3. The sequence $0 \rightarrow A \rightarrow B \stackrel{\pi}{\rightarrow} C \rightarrow 0$ is ch-pure if and only if $0 \rightarrow A \rightarrow B \rightarrow C \rightarrow 0$ is balanced and the map $\pi: p^{\lambda} c(B) \rightarrow p^{\lambda} c(C)$ is surjective for every limit ordinal $\lambda$ such that $\lambda$ is not cofinal with $\omega$.

Proof. Suppose that the sequence $e: 0 \rightarrow A \rightarrow B \stackrel{\pi}{\rightarrow} C \rightarrow 0$ is balanced. We may assume that $A, B$ and $C$ are reduced and submodules of $c(A), c(B)$ and $c(C)$ respectively. To show that the sequence $e$ is ch-pure it will be sufficient to show that for every ordinal $\alpha$ the map $\pi: p^{\alpha} c(B) \rightarrow p^{\alpha} c(C)$ is surjective. Let $\alpha=\lambda+n$ where $\lambda$ is a limit ordinal and $0 \leqslant n<\omega$.

Case I. If $\lambda$ is not cofinal with $\omega$ then the map $\pi: p^{\lambda} c(B) \rightarrow p^{\lambda} c(C)$ being surjective implies that the map $\pi: p^{\alpha} c(B) \rightarrow p^{\alpha} c(C)$ is surjective.

Case II. Suppose $\lambda$ is cofinal with $\omega$. Let $M$ be a $\lambda$-elementary balanced projective and $m$ a generator of the free $Z_{p}$-module $p^{\alpha} M$. If $c \in p^{\alpha} c(C)$ then there 
is a homomorphism $f: M \rightarrow c(C)$ such that $f(m)=c$. Let $S$ be the torsion submodule of $M$ and let $f^{\prime}$ be the restriction of $f$ to $S$. Thus $f^{\prime}$ is a homomorphism from $S$ to $C$. Since $\lambda$ is cofinal with $\omega, S$ is a totally projective $p$-group and there is a homomorphism $g: S \rightarrow B$ such that $\pi g=f^{\prime}$. The homomorphism $g$ lifts to a homomorphism $g^{\prime}: M \rightarrow c(B)$ since $c(B)$ is cotorsion and $M / S$ is torsion-free. Since $M / S$ is torsion-free and divisible, $\pi g^{\prime}=f: M \rightarrow c(C)$. Thus $g^{\prime}(m) \in p^{\alpha} c(B)$ and $\pi\left(g^{\prime}(m)\right)=c$. This implies that $\pi: p^{\alpha} c(B) \rightarrow p^{\alpha} c(C)$ is surjective.

Another interesting characterization of ch-purity is the following:

THEOREM 2.4. A sequence $0 \rightarrow A \stackrel{\iota}{\rightarrow} B \stackrel{\pi}{\rightarrow} C \rightarrow 0$ is ch-pure if and only if $0 \rightarrow A \rightarrow$ $B \rightarrow C \rightarrow 0$ is balanced and the sequence $0 \rightarrow K\left(p^{\lambda}, A\right) \stackrel{\iota}{\rightarrow} K\left(p^{\lambda}, B\right) \stackrel{\pi}{\rightarrow} K\left(p^{\lambda}, C\right) \rightarrow 0$ is exact for every limit ordinal $\lambda$ such that $\lambda$ is not cofinal with $\omega$.

Proof. Suppose that the sequence $0 \rightarrow A \rightarrow B \rightarrow C \rightarrow 0$ is ch-pure. Then the following commutative diagram has exact rows and columns. ( $\lambda$ is any ordinal).

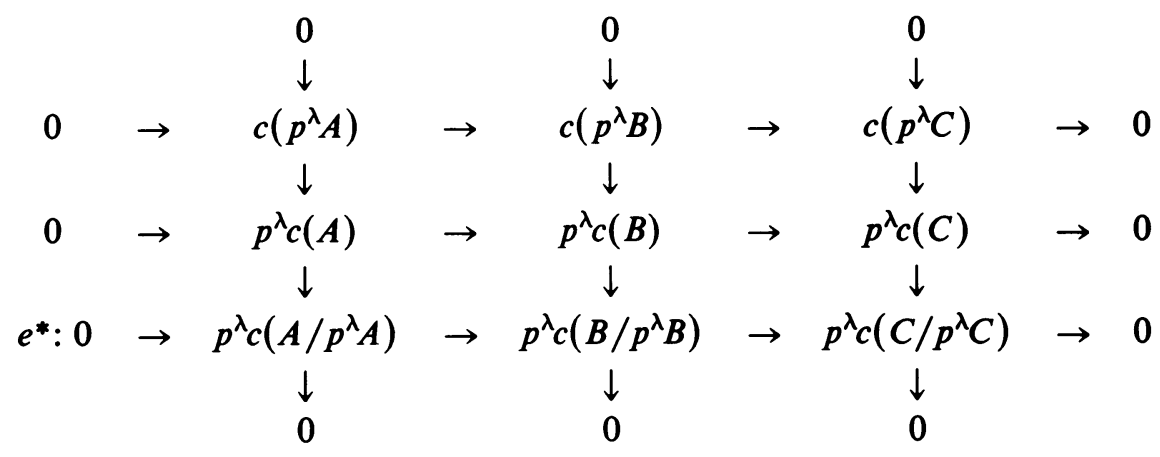

Since $p^{\lambda} c\left(C / p^{\lambda} C\right)$ is torsion-free, the sequence $e^{*}$ is pure exact. This implies that the sequence

$$
0 \rightarrow K\left(p^{\lambda}, A\right) \rightarrow K\left(p^{\lambda}, B\right) \rightarrow K\left(p^{\lambda}, C\right) \rightarrow 0
$$

is exact.

To prove the converse, it will be sufficient to show that $\pi: p^{\lambda} c(B) \rightarrow p^{\lambda} c(C)$ is surjective for every limit ordinal $\lambda$ such that $\lambda$ is not cofinal with $\omega$. Let $X_{0}$ be a basis for $K\left(p^{\lambda}, A\right)$. Since the sequence $0 \rightarrow K\left(p^{\lambda}, A\right) \stackrel{\iota}{\rightarrow} K\left(p^{\lambda}, B\right) \stackrel{\pi}{\rightarrow} K\left(p^{\lambda}, C\right) \rightarrow 0$ is exact, the image of $X_{0}, \iota\left(X_{0}\right)$, in $K\left(p^{\lambda}, B\right)$ can be extended by a set $X$ such that $\iota\left(X_{0}\right) \cup X$ is a basis for $K\left(p^{\lambda}, B\right)$ and $\pi(X)$ is a basis for $K\left(p^{\lambda}, C\right)$.

Let $X_{0}$ and $X$ be subsets of $p^{\lambda} c(B)$ which are mapped bijectively by the natural map $\sigma_{\lambda}: p^{\lambda} c(B) \rightarrow p^{\lambda} c\left(B / p^{\lambda} B\right) / p^{\lambda+1} c\left(B / p^{\lambda} B\right)=K\left(p^{\lambda}, B\right)$ onto $\iota\left(X_{0}\right)$ and $X$ respectively. There is a torsion-free submodule $L$ of $p^{\lambda} c(B)$ such that $p^{\lambda} c(B)=c\left(p^{\lambda} B\right) \oplus$ $L$ where $L$ is the $p$-adic completion of the free module generated by $\underline{X}_{0} \cup \underline{X}$. Similarly there is a submodule $L^{\prime}$ of $p^{\lambda} c(C)$ such that $p^{\lambda} c(C)=c\left(p^{\lambda} C\right) \bar{\oplus}^{\prime}$ and $L^{\prime}$ is the $p$-adic completion of the free module generated by $\pi(X)$. Clearly there is a surjective homomorphism $\gamma: L \rightarrow L^{\prime}$ such that for each $x \in \bar{X}, \gamma(x)=\pi(x)$. Since the sequence $0 \rightarrow p^{\lambda} A \rightarrow p^{\lambda} B \rightarrow p^{\lambda} C \rightarrow 0$ is exact, $\pi: c\left(p^{\lambda} B\right) \rightarrow c\left(p^{\lambda} C\right)$ is surjective. Thus a homomorphism $\gamma^{\prime}: p^{\lambda} c(B) \rightarrow p^{\lambda} c(C)$ can be constructed such that $\gamma^{\prime}$ is surjective and $\gamma^{\prime}$ agrees with $\pi$ on the submodule $c\left(p^{\lambda} B\right)+\left[\underline{X}_{0} \cup \underline{X}\right]$. Since 
$p^{\lambda} c(B) /\left(c\left(p^{\lambda} B\right)+\left[\underline{X}_{0} \cup \underline{X}\right]\right)$ is torsion-free and divisible, $\pi=\gamma^{\prime}$ and $\pi: p^{\lambda} c(B) \rightarrow$ $p^{\lambda} c(C)$ is surjective.

THEOREM 2.5. If $N$ is a $Z_{p}$-module then there is a ch-pure sequence $e: 0 \rightarrow A \rightarrow B$ $\rightarrow N \rightarrow 0$ such that $B$ is the direct sum of a divisible module and an SKT-module; i.e., $B$ is a ch-projective module. If $N$ is reduced then $B$ can be chosen to be an SKT-module.

Proof. Let $N=D \oplus R$ where $D$ is the maximal divisible submodule of $N$ and $R$ is a reduced module. There are balanced sequences $0 \rightarrow A_{1} \rightarrow P_{1} \rightarrow R \stackrel{\pi_{1}}{\rightarrow} 0$ and $0 \rightarrow A_{2} \rightarrow P_{2} \stackrel{\pi_{2}}{\rightarrow} c(R) \rightarrow 0$ such that $P_{1}$ and $P_{2}$ are balanced projectives. Let $S$ be the torsion subgroup of $P_{2}, B=D \oplus P_{1} \oplus S$ and define $\pi: B \rightarrow N$ by $\pi(d, p, s)=$ $d+\pi_{1}(p)+\pi_{2}(s)$. If $A$ is the kernel of $\pi$ then clearly the sequence $0 \rightarrow A \rightarrow$ $B \stackrel{\pi}{\rightarrow} N \rightarrow 0$ is balanced; thus we need only demonstrate that for the ordinal $\alpha$, the map $\pi: p^{\alpha} c(B) \rightarrow p^{\alpha} c(N)$ is surjective. In fact, it may be assumed that $\alpha$ is an infinite ordinal. The injection map $\iota: S \rightarrow P_{2}$ induces the isomorphism $\iota: p^{\alpha} c(S) \rightarrow$ $p^{\alpha} c\left(P_{2}\right)$. Consequently, the map $\pi_{2}: p^{\alpha} c(S) \rightarrow p^{\alpha} c(R) \cong p^{\alpha} c(N)$ is surjective. This implies that the map $\pi: p^{\alpha} c(B) \rightarrow p^{\alpha} c(N)$ is surjective.

THeOREM 2.6. If $M$ is a $Z_{p}$-module and $\alpha$ an ordinal then $M$ is ch-projective if and only if $p^{\alpha} M$ and $M / p^{\alpha} M$ are ch-projective.

Proof. Clearly if $M$ is ch-projective then $p^{\alpha} M$ and $M / p^{\alpha} M$ are ch-projective.

Suppose $p^{\alpha} M$ and $M / p^{\alpha} M$ are ch-projective and $e: 0 \rightarrow A \rightarrow B \stackrel{\pi}{\rightarrow} C \rightarrow 0$ is a ch-pure sequence. First it will be shown that the sequence $e_{\alpha}: 0 \rightarrow p^{\alpha} A \rightarrow$ $p^{\alpha} B \stackrel{\pi}{\rightarrow} p^{\alpha} C \rightarrow 0$ is ch-pure. Clearly the sequence $e_{\alpha}$ is balanced. Thus to show that $e_{\alpha}$ is ch-pure it is required only to show that the map $\pi: p^{\lambda} c\left(p^{\alpha} B\right) \rightarrow p^{\lambda} c\left(p^{\alpha} C\right)$ is surjective for all limit ordinals $\lambda>0$ such that $\lambda$ is not cofinal with $\omega$. There are torsion-free modules $X$ and $Y$ such that $p^{\alpha} c(B)=c\left(p^{\alpha} B\right) \oplus X$ and $p^{\alpha} c(C)=$ $c\left(p^{\alpha} C\right) \oplus Y$. Since $\lambda$ is an infinite ordinal, $p^{\lambda} p^{\alpha} c(B)=p^{\lambda} c\left(p^{\alpha} B\right)$ and $p^{\lambda} p^{\alpha} c(C)=$ $p^{\lambda} c\left(p^{\alpha} B\right)$. It follows that the map $\pi: p^{\lambda} c\left(p^{\alpha} B\right) \rightarrow p^{\lambda} c\left(p^{\alpha} C\right)$ is surjective because the map $\pi: p^{\lambda} p^{\alpha} c(B) \rightarrow p^{\lambda} p^{\alpha} c(C)$ is surjective.

Let $f \in \operatorname{Hom}(M, C)$. Then $f\left(p^{\alpha} M\right) \subseteq p^{\alpha} C$. There exists a homomorphism $g$ : $p^{\alpha} M \rightarrow p^{\alpha} B$ because $p^{\alpha} M$ is ch-projective and the sequence $0 \rightarrow p^{\alpha} A \rightarrow p^{\alpha} B \rightarrow p^{\alpha} C$ $\rightarrow 0$ is ch-pure. The homomorphism $g$ lifts to a homomorphism $g^{*}: M \rightarrow B$. Let $h=f-\pi g^{*}: M \rightarrow C$ and note that $h\left(p^{\alpha} M\right)=0$. Consequently, we can consider $h$ as a homomorphism from $M / p^{\alpha} M$ to $C$. Since $M / p^{\alpha} M$ is ch-projective, there is a homomorphism $\gamma: M \rightarrow M / p^{\alpha} M \rightarrow B$ such that $\pi \gamma=h=f-\pi g^{*}$ or, equivalently, $f=\pi\left(\gamma+g^{*}\right)$. Thus $M$ is ch-projective.

\section{REFERENCES}

1. R. B. Warfield, Jr., A classification theorem for Abelian p-groups, Trans. Amer. Math. Soc. 210 (1975), 149-168.

2. Classification theory of Abelian groups. I: Balanced projectives, Trans. Amer. Math. Soc. 222 (1976), 33-63. 